\title{
On absolutely normal and continued fraction normal numbers
}

\author{
Verónica Becher Sergio A. Yuhjtman
}

March 5, 2018

\begin{abstract}
We give a construction of a real number that is normal to all integer bases and continued fraction normal. The computation of the first $n$ digits of its continued fraction expansion performs in the order of $n^{4}$ mathematical operations. The construction works by defining successive refinements of appropriate subintervals to achieve, in the limit, simple normality to all integer bases and continued fraction normality. The main difficulty is to control the length of these subintervals. To achieve this we adapt and combine known metric theorems on continued fractions and on expansions in integers bases.
\end{abstract}

For a real number $x$ in the unit interval, the continued fraction expansion of $x$ is a sequence of positive integers $a_{1}, a_{2}, \ldots$, such that

$$
x=\frac{1}{a_{1}+\frac{1}{a_{2}+\frac{1}{\ddots+\frac{1}{a_{n}+\frac{1}{\ddots}}}}}
$$

A real number is continued fraction normal if every block of integers occurs in the continued fraction expansion with the asymptotic frequency determined by the Gauss measure. An application of Birkhoff's Ergodic Theorem proves that almost all -with respect to Lebesgue measure- real numbers have a normal continued fraction expansion.

For each real number $x$ in the unit interval, its expansion in an integer base $b$ greater than or equal to 2 is a sequence of integers $a_{1}, a_{2} \ldots$, where $0 \leq a_{i}<b$ for every $i$, such that

$$
x=\sum_{i=1}^{\infty} a_{i} b^{-i} .
$$

We require that $a_{i}<b-1$ infinitely many times to ensure that every rational number has a unique representation. A real number $x$ is simply normal to a given base $b$ if every digit occurs in the $b$-ary expansion of $x$ with the same asymptotic frequency. Normality to base $b$ is defined as simple normality to $b, b^{2}, b^{3}, \ldots$, all the powers of $b$. In 1940 Pillai proved that this formulation of normality is equivalent to Borel's original definition in [4] (see [5, Theorem 4.2]). Absolute normality is defined as normality to every integer base greater than or equal to 2 , hence, as simple normality to every integer base greater than or equal to 2 . Borel showed that almost all (with respect to Lebesgue measure) real numbers are absolutely normal. 
Thus, almost all real numbers are absolutely normal and continued fraction normal. Here we prove the following:

Theorem 1. There is an algorithm that computes a number that is absolutely normal and continued fraction normal. The computation of the first $n$ digits of the continued fraction expansion performs a number of mathematical operations that is in $O\left(n^{4}\right)$.

On the problem of constructing a number satisfying the two forms of normality. The problem appeared explicitly in the literature first in [16] and then in [5. Problem 10.49]. Recently Scheerer [17] gave an algorithm that yields one such number with doubly exponential computational complexity: the computation of the first $n$ digits of the continued fraction expansion performs doubly exponential in $n$ many mathematical operations. Thus, as any other algorithm with exponential complexity, Scheerer's algorithm can not run in human time. In contrast, algorithms with polynomial complexity can. To prove Theorem 1 we give an algorithm that can be implemented as an efficient computer program that outputs one digit after the other. Our technique elaborates on the algorithm given by Becher, Heiber and Slaman [2] which has just above quadratic complexity. Madritsch, Scheerer and Tichy [10] also elaborated on the algorithm given in [2], but in a very different way, and they use it to compute absolutely normal Pisot numbers efficiently. Finally we remark that if in our algorithm we skip the treatment of integer bases then we obtain a continued fraction normal number (with no guarantee of normality to integer bases). Such an algorithm differs substantially from all the previously known constructions of continued fraction normal numbers [15, 1, 15, 11, 17].

About the proof of Theorem 1. Our algorithm works incrementally to define, in the limit of the computation, a real number $x$ in the unit interval. The construction works by defining successive refinements of appropriate subintervals to achieve, in the limit, simple normality to all integer bases and continued fraction normality. The choice of each subinterval determines further digits in the expansion of $x$ in integer bases and in its continued fraction. We require that the choice contributes to the two forms of normality but without revisiting the previous digits. For this we need to control, at each step of the construction, the lengths of the new subintervals, as they should be not too small.

We say that an interval $I$ is $c f$-ary if there is a finite continued fraction $\left[a_{1}, \ldots, a_{n}\right]$ such that the interval $I$ is equal to the set of all the numbers whose first $n$ digits of their continued fraction expansion are $a_{1}, \ldots, a_{n}$. And we say that an interval $J$ is $b$-ary for some integer $b$ greater than or equal to 2 if there is a finite block $d_{1}, \ldots, d_{n}$ of digits between 0 and $b-1$ such that $J$ is equal to the set of real numbers whose first $n$ digits of their $b$-ary expansion are equal to $d_{1}, \ldots, d_{n}$. The set of $b$-ary intervals determined by $n$ digits between 0 and $b-1$ is a partition of the unit interval in finitely many parts of equal length. The set of $c f$-ary intervals determined by $n$ digits also form a partition of the unit interval, but in infinite parts of different length. Our construction rests on the fact that the $c f$-ary intervals determined by any $n$ digits have, nevertheless, an expected approximate length. This is because the distribution of the logarithm of the convergent of finite continued fractions of $n$ digits obeys a Gaussian law. Ibragimov [8] was the first to establish this theorem. Morita [13, Theorem 8.1] and Vallée [18, Theorem 9] obtained the same result with an optimal error term. We use this optimal version in Lemma 5 to guarantee the existence of enough $c f$-ary subintervals having the desired relative length with respect to the previously considered $c f$-ary interval. The control of the length of the $b$-ary subintervals is much simpler. Proposition 12 gives the needed estimate for the relative size of a $b$-ary subinterval of any given interval. 
To achieve continued fraction normality we need to bound the measure of the set inside any given $c$-ary interval $I$ having too many or too few occurrences of a given block of digits in their continued fraction expansions. This is essentially a result on large deviations proved by Kifer, Peres and Weiss [9, Corollary 3.2] but conditioned on the first digits that determine the interval $I$. We establish this result in Lemma 3. To achieve normality in each integer base $b$ we use Hardy and Wright's estimate [7, Proof of Theorem 148] for the number of blocks having too many or too few occurrences of a given digit, as stated in Lemma 8 . At each step of the computation the algorithm determines a finite extension of the continued fraction expansion of $x$, as well as a finite extension of the expansion of $x$ in each of the finitely many integer bases designated for that step. To obtain absolute normality the set of designated integer bases increases with the step number, and in the limit it consists of all integers greater than or equal to 2. To obtain continued fraction normality, at each step the algorithm considers the occurrences of blocks from certain finite collection. The set of designated blocks increases monotonically in the step number and in the limit consists of all blocks of all positive integers.

Organisation of the paper. We devote Section 1 to the definitions and the tools to be used in the proof of Theorem 1. We first present a non-recursive formulation of the convergent of a finite continued fraction and we use it in Lemma 3 to obtain convenient upper and lower bounds of the length of any $c f$-ary subinterval of a given $c f$-ary interval. These upper and lower bounds propagate along most of the results of this work. We give aforementioned key Lemmas 5, 6 and 8, as well as the material to deal with the discrepancy associated to continued fraction expansions and $b$-ary expansions, see Lemmas 7 and 9. In Section 2 we give the actual proof of Theorem 1. We present the algorithm, we prove its correctness and we estimate the number of mathematical operations needed to compute the first $n$ digits of the continued fraction expansion of the number defined by our algorithm. The algorithm and its correctness are based on Lemma [13, which is the main lemma of the paper.

\section{Needed definitions and lemmas}

Notation. As usual we write $\mathbb{N}$ to denote the set of positive integers and $\mathbb{N}^{k}$ to denote the set of $k$ tuples of positive integers. For a finite set $S$, \#S is its cardinality. For an infinite set $S$ of real numbers, $|S|$ is its Lebesgue measure; hence, when $S$ is an interval, $|S|$ is its length. We use standard notation for the asymptotic behaviour of functions. We say that a function $g(x)$ is $O(f(x))$ if there are constants $x_{0}$ and $c$ such that for every $x \geq x_{0},|g(x)|<c \cdot|f(x)|$. We write log to denote the logarithm in base $e$.

\section{$1.1 \quad c f$-ary intervals}

We write $x=\left[a_{1}, a_{2}, \ldots\right]$ with $a_{1}, a_{2}, \ldots$ positive integers to denote the continued fraction expansion of a real number $x$ in the unit interval. The functions $p_{n}(x)$ and $q_{n}(x)$, called the convergents of $x$, are defined recursively as follows.

$$
\begin{aligned}
& \text { If } x=\left[a_{1}, a_{2}, \ldots\right], \\
& \qquad \begin{array}{l}
p_{-1}(x)=q_{0}(x)=1 \\
p_{0}(x)=q_{-1}(x)=0
\end{array}
\end{aligned}
$$


and for $n \geq 1$,

$$
\begin{aligned}
& p_{n}(x)=a_{n} p_{n-1}(x)+p_{n-2}(x), \\
& q_{n}(x)=a_{n} q_{n-1}(x)+q_{n-2}(x) .
\end{aligned}
$$

When the real number $x$ is understood from the context we write $p_{-1}, q_{-1}, p_{0}, q_{0}, \ldots$ instead of $p_{-1}(x), q_{-1}(x), p_{0}(x), q_{0}(x), \ldots$ Given $a_{1}, \ldots, a_{n}$, we have the equation

$$
\left[a_{1}, \ldots, a_{n}\right]=\frac{p_{n}}{q_{n}} .
$$

For irrational $x=\left[a_{1}, a_{2}, \ldots\right], p_{n} / q_{n}$ is the $n$th approximant to $x$ and converges to $x$ as $n$ tends to infinity. For rational $x=\left[a_{1}, \ldots, a_{n}\right], x=p_{n} / q_{n}$. Observe that $\left(p_{n}\right)_{n \geq 1}$ and $\left(q_{n}\right)_{n \geq 1}$ are increasing.

The convergent $q_{n}$ can be expressed by a non-recursive formula, as follows. We write $\amalg$ to denote the disjoint union operation. We define the set $\mathcal{P}$ of subsets of positive integers as

$$
\mathcal{P}=\left\{C \subset \mathbb{N}:(\exists D \subset \mathbb{N}) C=\amalg_{n \in D}\{n, n+1\}\right\} .
$$

For every pair of positive integers $r, s$ such that $r \leq s$, define

$$
\begin{aligned}
\Omega_{r, s} & =\{\mathcal{I} \subset\{r, \ldots, s\}:(\{r, \ldots, s\} \backslash \mathcal{I}) \in \mathcal{P}\} . \\
\alpha_{r, s} & =\sum_{\mathcal{I} \in \Omega_{r, s}} \prod_{i \in \mathcal{I}} a_{i} .
\end{aligned}
$$

And let

$$
\alpha_{s+1, s}=1 \text { and } \alpha_{s+2, s}=0 .
$$

The following proposition holds.

Proposition 2. Let $x=\left[a_{1}, a_{2}, \ldots\right] \in(0,1)$.

1. For every positive integer $s, q_{s}(x)=\alpha_{1, s}$.

2. Let $r, r^{\prime}, s, s^{\prime}$ be positive integers. If $r \leq r^{\prime}$ and $s \geq s^{\prime}$ then $\alpha_{r, s} \leq \alpha_{r^{\prime}, s^{\prime}}$. Equality holds if and only if $r=r^{\prime}$ and $s=s^{\prime}$.

3. Let $r$ and $s$ be positive integers. If $r \leq s$ then $\alpha_{1, s}=\alpha_{1, r} \alpha_{r+1, s}+\alpha_{1, r-1} \alpha_{r+2, s}$.

Proof. Item 1 is true for $s=1$ and it follows by induction from $q_{n}=a_{n} q_{n-1}+q_{n-2}$. Items 2 and 3 follow from the definition of $\alpha_{r, s}$.

For a finite continued fraction $\left[a_{1}, \ldots, a_{n}\right]$ we consider the open interval $I_{\left[a_{1}, \ldots, a_{n}\right]}$ containing the numbers whose first $n$ digits of their continued fraction expansion are $a_{1}, \ldots, a_{n}$. Thus,

$$
\begin{aligned}
& I_{\left[a_{1}, \ldots, a_{n}\right]}=\left(\left[a_{1}, \ldots, a_{n}\right],\left[a_{1}, \ldots, a_{n}+1\right]\right), \text { or } \\
& I_{\left[a_{1}, \ldots, a_{n}\right]}=\left(\left[a_{1}, \ldots, a_{n}+1\right],\left[a_{1}, \ldots, a_{n}\right]\right)
\end{aligned}
$$

We say that an interval $I$ is $c f$-ary of order $n$ if it is some $I_{\left[a_{1}, \ldots, a_{n}\right]}$.

The length of a $c f$-ary interval is

$$
\left|I_{\left[a_{1}, \ldots, a_{n}\right]}\right|=\frac{1}{q_{n}\left(q_{n}+q_{n-1}\right)} .
$$

If $a=\left[a_{1}, \ldots, a_{r}\right], b=\left[a_{r+1}, \ldots, a_{s}\right]$ and $c=\left[a_{1}, \ldots, a_{s}\right]$, we write $I_{a, b}$ to denote $I_{c}$. For $x=\left[d_{1}, \ldots, d_{n}\right]$ we simply write $q(x)$ to denote $q_{n}(x)$. 
Lemma 3. Let $a=\left[a_{1}, \ldots, a_{r}\right], b=\left[a_{r+1}, \ldots, a_{s}\right]$ and $c=\left[a_{1}, \ldots, a_{s}\right]$. Then,

1. $q(a) q(b) \leq q(c) \leq 2 q(a) q(b)$.

2. $\left|I_{b}\right| / 2 \leq\left|I_{a, b}\right| /\left|I_{a}\right| \leq 2\left|I_{b}\right|$.

Proof. 1. Using Proposition 2.

$$
\begin{aligned}
q(a) q(b) & =\alpha_{1, r} \alpha_{r+1, s} \leq \alpha_{1, r} \alpha_{r+1, s}+\alpha_{1, r-1} \alpha_{r+2, s}=\alpha_{1, s}=q(c) . \\
q(c) & =\alpha_{1, r} \alpha_{r+1, s}+\alpha_{1, r-1} \alpha_{r+2, s} \leq 2 \alpha_{1, r} \alpha_{r+1, s} \leq 2 q(a) q(b) .
\end{aligned}
$$

2. $\left|I_{a, b}\right|^{-1}=\alpha_{1, s}\left(\alpha_{1, s}+\alpha_{1, s-1}\right)$

$$
\begin{aligned}
& =\left(\begin{array}{ll}
\alpha_{1, r} & \alpha_{r+1, s}+\alpha_{1, r-1} \\
\alpha_{r+2, s}
\end{array}\right)\left(\alpha_{1, r}\left(\alpha_{r+1, s}+\alpha_{r+1, s-1}\right)+\alpha_{1, r-1}\left(\alpha_{r+2, s}+\alpha_{r+2, s-1}\right)\right) \\
& \leq 2 \alpha_{1, r} \alpha_{r+1, s}\left(\alpha_{1, r}\left(\alpha_{r+1, s}+\alpha_{r+1, s-1}\right)+\alpha_{1, r-1}\left(\alpha_{r+1, s}+\alpha_{r+1, s-1}\right)\right) \\
& =2 \alpha_{1, r}\left(\alpha_{1, r}+\alpha_{1, r-1}\right) \alpha_{r+1, s}\left(\alpha_{r+1, s}+\alpha_{r+1, s-1}\right) \\
& =2\left|I_{a}\right|^{-1}\left|I_{b}\right|^{-1} \text {. }
\end{aligned}
$$

$$
\begin{aligned}
\left|I_{a, b}\right|^{-1} & =\alpha_{1, s}\left(\alpha_{1, s}+\alpha_{1, s-1}\right) \\
& =\left(\alpha_{1, r} \alpha_{r+1, s}+\alpha_{1, r-1} \alpha_{r+2, s}\right)\left(\alpha_{1, r}\left(\alpha_{r+1, s}+\alpha_{r+1, s-1}\right)+\alpha_{1, r-1}\left(\alpha_{r+2, s}+\alpha_{r+2, s-1}\right)\right) \\
& \geq\left(\alpha_{1, r} \alpha_{r+1, s}\right)\left(\alpha_{1, r}\left(\alpha_{r+1, s}+\alpha_{r+1, s-1}\right)\right) \\
& \geq\left(\alpha_{1, r} \alpha_{r+1, s}\right)\left(\frac{\alpha_{1, r}+\alpha_{1, r-1}}{2}\right)\left(\alpha_{r+1, s}+\alpha_{r+1, s-1}\right) \\
& =\frac{1}{2}\left|I_{a}\right|^{-1}\left|I_{b}\right|^{-1} .
\end{aligned}
$$

The distribution of $\log q_{n}$ obeys in the limit a Gaussian law. It was first proved by Ibragimov [8]. Then Philipp [14, Satz 3] obtained an error term of $O\left(n^{-1 / 5}\right)$, which was later improved by Mischyavichyus [12 to $O\left(n^{-1 / 2} \log n\right)$. Morita [13, Theorem 8.1] obtained the optimal error term of order $O\left(n^{-1 / 2}\right)$; a different proof of the same bound was given by Vallée [18, Théoreme 9]. In the sequel we write $L$ for Lévy's constant $\pi^{2} /(12 \log 2)$.

Lemma 4 (Morita [13, Theorem 8.1], Vallée [18, Théoreme 9]). The distribution of the random variable $\log q_{n}(x)$ is asymptotically Gaussian. There is $K_{0}$ and $n_{0}$ such that for every $n \geq n_{0}$,

$$
\left|\operatorname{Pr}\left[x \in(0,1):-y \leq \frac{\log q_{n}(x)-n L}{\sigma \sqrt{n}} \leq y\right]-\frac{1}{\sqrt{2 \pi}} \int_{-y}^{y} e^{-z^{2} / 2} d z\right|<\frac{K_{0}}{\sqrt{n}},
$$

where $\sigma$ is a positive absolute constant.

Vallée in [18] and also in [6], obtained an expression for $\sigma$ using the generalised transfer operators $L_{s}$ for $s>1$ over a suitable space of functions, also known as the Ruelle-Mayer operator, defined by

$$
L_{s}[f](z)=\sum_{n=1}^{\infty}\left(\frac{1}{n+z}\right)^{s} f\left(\frac{1}{n+z}\right)
$$


These operators $L_{s}$ have a simple dominant positive eigenvalue $\lambda(s)$. The expression for $\sigma$ uses the dominant eigenvalue of $L_{2}$,

$$
\sigma^{2}=\lambda^{\prime \prime}(2)-\lambda^{\prime}(2)^{2}
$$

where $\lambda^{\prime}$ and $\lambda^{\prime \prime}$ denote the derivative and second derivative of $\lambda$ and

$\lambda^{\prime}(2)=-\pi^{2} /(12 \log 2)$ is Levy's constant with negative sign.

$\lambda^{\prime \prime}(2)$ is the variance of the law of continuants, known as Hensley's constant.

We remark that our use of $\sigma$ occurs just in Lemma 5 and we not require its exact value $\sigma$, any upper bound suffices.

Lemma 5. There are positive constants $K, c$ and a positive integer $n_{1}$ such that for any cf-ary interval $I$ and any integer $n \geq n_{1}$, the Lebesgue measure of the union of the cf-ary subintervals $J$ of $I$ of relative order $n$ such that

$$
\frac{|I|}{4} e^{-2 n L-2 c} \leq|J| \leq 2|I| e^{-2 n L+2 c}
$$

is greater than $K|I| / \sqrt{n}$.

Proof. Take a positive constant $c>\sigma \sqrt{2 \pi} K_{0}$, say $c=2 \sigma \sqrt{2 \pi} K_{0}$. For each positive integer $n$ consider the set

$$
S_{n}=\left\{x \in(0,1):-c \leq \log q_{n}(x)-n L \leq c\right\}
$$

By Lemma 4 there is $K_{0}$ and $n_{0}$ such that for every integer $n \geq n_{0}$, the Lebesgue measure of $S_{n}$ is at least

$$
\frac{1}{\sqrt{2 \pi}} \int_{-c /(\sigma \sqrt{n})}^{c /(\sigma \sqrt{n})} e^{-w^{2} / 2} d w-\frac{K_{0}}{\sqrt{n}}
$$

But for $n$ sufficiently large so that $e^{-w^{2} / 2}>1 / 2$ in the following integration region,

$$
\frac{1}{\sqrt{2 \pi}} \int_{-c /(\sigma \sqrt{n})}^{c /(\sigma \sqrt{n})} e^{-w^{2} / 2} d w>\frac{1}{\sqrt{2 \pi}} \frac{c}{\sigma \sqrt{n}}=2 \frac{K_{0}}{\sqrt{n}}
$$

hence we have that the Lebesgue measure of $S_{n}$ is at least $K_{0} / \sqrt{n}$.

Consider a sequence of digits $a_{1}, a_{2}, \ldots a_{n}$ such that

$$
-c \leq \log q_{n}(x)-n L \leq c .
$$

This is equivalent to

$$
e^{-n L-c} \leq \frac{1}{q_{n}(x)} \leq e^{-n L+c} .
$$

We call $I_{n}=I_{a_{1}, \ldots, a_{n}}$, whose length is $\left|I_{n}\right|=1 /\left(q_{n}\left(q_{n}+q_{n-1}\right)\right)$. Clearly

$$
\frac{1}{2 q_{n}^{2}} \leq\left|I_{n}\right| \leq \frac{1}{q_{n}^{2}}
$$

Fix a $c f$-ary interval $I$. The concatenation of $a_{1}, \ldots, a_{n}$ after the digits that define $I$ yields a $c f$-ary subinterval $J$ of $I$. By Lemma 3 ,

$$
\frac{1}{4 q_{n}^{2}} \leq \frac{\left|I_{n}\right|}{2} \leq \frac{|J|}{|I|} \leq 2\left|I_{n}\right| \leq \frac{2}{q_{n}^{2}}
$$


Thus,

$$
\frac{1}{4}|I| e^{-2 n L-2 c} \leq|J| \leq 2|I| e^{-2 n L-2 c} .
$$

Then, the set of $c f$-ary subintervals $J$ of $I$ of relative order $n$ such that

$$
\frac{|I|}{4} e^{-2 n L-2 c} \leq|J| \leq 2|I| e^{-2 n L+2 c}
$$

has Lebesgue measure at least

$$
K_{0} /(2|I| \sqrt{n})
$$

\subsection{Discrepancy associated to continued fraction expansions}

The Gauss map $T$ is a function from real numbers in the unit interval to real numbers in the unit interval, defined by $T(0)=0$ and $T(x)=1 / x-\lfloor 1 / x\rfloor$. If $\left[a_{1}, a_{2}, \ldots\right]$ denotes the continued fraction expansion of $x$, then $T^{n}(x)=\left[a_{n+1}, a_{n+2}, \ldots\right]$ and $a_{n}=\left\lfloor 1 / T^{n-1}(x)\right\rfloor$, for $n \geq 1$. The map $T$ possesses an invariant ergodic measure, the Gauss measure $\mu$, which is absolutely continuous with respect to Lebesgue measure,

$$
\mu(\mathrm{d} x)=\frac{\mathrm{d} x}{(1+x) \log 2} .
$$

Then the Gauss measure for a $c f$-ary interval $I_{\left[a_{1}, \ldots, a_{n}\right]}$ in the unit interval is,

$$
\mu\left(I_{\left[a_{1}, \ldots, a_{n}\right]}\right)=\int_{r / s}^{r^{\prime} / s^{\prime}} \mu(\mathrm{d} x),
$$

where $r / s$ and $r^{\prime} / s^{\prime}$ denote the rational numbers $\left[a_{1}, \ldots, a_{n}\right]$ and $\left[a_{1}, \ldots, a_{n}+1\right]$ ordered such that $r / s<r^{\prime} / s^{\prime}$.

We write $\mathbb{I}_{I}(x)$ to denote the characteristic function of the interval $I$, so $\mathbb{I}_{I}(x)=1$ if $x \in I$ and $\mathbb{I}_{I}(x)=0$, otherwise. We say that $\left[a_{1}, a_{2}, \ldots\right]$ is a normal continued fraction if, for every positive integer $k$ and for every block of $k$ positive integers $v_{1}, \ldots, v_{k}$,

$$
\lim _{n \rightarrow \infty} \frac{1}{n} \sum_{j=0}^{n-1} \mathbb{I}_{\left[v_{1}, \ldots, v_{k}\right]}\left(T^{j} x\right)=\mu\left(I_{\left[v_{1}, \ldots, v_{k}\right]}\right) .
$$

Equivalently,

$$
\lim _{n \rightarrow \infty} \frac{1}{n} \#\left\{j: 1 \leq j \leq n, a_{j}=v_{1}, \ldots, a_{j+k-1}=v_{k}\right\}=\mu\left(I_{\left[v_{1}, \ldots, v_{k}\right]}\right) .
$$

For example, quadratic irrationals do not have a normal continued fraction expansion because they are periodic. The continued fraction expansion of $e,[2 ; 1,2,1,1,4,1,1,6,1,1,8, \ldots]$, is not normal either because it is the concatenation of the pattern $(1 \mathrm{~m} 1)$, for all even $m$ in increasing order. Applying Birkhoff's Ergodic Theorem [3] we obtain that almost every real in the unit interval has normal continued fraction expansion. 
For a real number $x$ and a block $v$ of $k$ positive integers $v_{1}, \ldots, v_{k}$ the discrepancy of $x$ with respect to $v$ in the first $n$ positions of its continued fraction expansion is defined as

$$
D_{v, n}^{c f-a r y}(x)=\left|\frac{1}{n} \sum_{j=0}^{n-1} \mathbb{I}_{\left[v_{1}, \ldots, v_{k}\right]}\left(T^{j} x\right)-\mu\left(I_{\left[v_{1}, \ldots, v_{k}\right]}\right)\right| .
$$

Clearly, a real number $x$ has a normal continued fraction if and only if for every positive integer $k$, and for every block $v$ of $k$ positive integers,

$$
\lim _{n \rightarrow \infty} D_{v, n}^{c f-a r y}(x)=0 .
$$

The following result on large deviations is essentially Kifer, Peres and Weiss' Corollary 3.2 in [9] but conditioning on the first $r$ terms.

Lemma 6. Let $I_{\left[a_{1}, \ldots, a_{r}\right]}$ be a cf-ary interval, and let $b$ be a block of $k$ positive integers $b_{1}, \ldots, b_{k}$. Then for every positive real $\delta$ and for every positive integer $n$,

$$
\left|\left\{x \in I_{\left[a_{1}, \ldots, a_{r}\right]}:\left|\frac{1}{n} \sum_{i=0}^{n-1} \mathbb{I}_{\left[b_{1}, \ldots, b_{k}\right]}\left(T^{r+i} x\right)-\mu\left(I_{\left[b_{1}, \ldots, b_{k}\right]}\right)\right|>\delta\right\}\right| \leq 6 M e^{-\frac{\delta^{2} n}{2 M}}\left|I_{\left[a_{1} \ldots, a_{r}\right]}\right|,
$$

where $M=M(\delta, k)=\left\lceil k-\log \left(\delta^{2} /(2 \log 2)\right)\right]$, or any larger number.

Proof. We write $I_{a}$ and $I_{b}$ to denote, respectively, $I_{\left[a_{1}, \ldots, a_{r}\right]}, I_{\left[b_{1}, \ldots, b_{k}\right]}$. Define

$$
\begin{aligned}
& \tau_{b, n, \delta}=\left\{x \in(0,1):\left|\frac{1}{n} \sum_{i=0}^{n-1} \mathbb{I}_{I_{b}}\left(T^{i} x\right)-\mu\left(I_{b}\right)\right|>\delta\right\} \\
& \tau_{b, n, \delta}^{a}=\left\{x \in I_{a}:\left|\frac{1}{n} \sum_{i=0}^{n-1} \mathbb{I}_{I_{b}}\left(T^{r+i} x\right)-\mu\left(I_{b}\right)\right|>\delta\right\}
\end{aligned}
$$

The set $\tau_{b, n, \delta}^{a}$ is the disjoint union of $c f$-ary intervals $I_{a c}$ where $c$ belongs to some appropriate set $\mathcal{Z} \subset \mathbb{N}^{k+n-1}$. The set $\tau_{b, n, \delta}$ is the disjoint union of $I_{c}$ where $c \in \mathbb{N}^{k+n-1}$ belongs to the same $\mathcal{Z}$. Lemma 3.1 and Remark 5.1 both in [9] establish that

$$
\mu\left(\tau_{b, n, \delta}\right) \leq 2 M(\delta, k) e^{-\frac{\delta^{2} n}{2 M(\delta, k)}},
$$

where

$$
M(\delta, k)=\min \left\{m \in \mathbb{N}:(\log 2) 2^{-m+k} \leq \delta^{2} / 2\right\} .
$$

For each $c \in \mathbb{N}^{k+n-1}$, by Lemma 32 ,

$$
\frac{\left|I_{a c}\right|}{\left|I_{a}\right|} \leq 2\left|I_{c}\right|
$$

Adding all these inequalities for $c$ in $\mathcal{Z}$ and given the fact that for all measurable $S$,

$$
|S| \leq 2(\log 2) \mu(S)<\frac{3}{2} \mu(S),
$$

we obtain

$$
\frac{\left|\tau_{b, n, \delta}^{a}\right|}{\left|I_{a}\right|} \leq 2\left|\tau_{b, n, \delta}\right| \leq 3 \mu\left(\tau_{b, n, \delta}\right) \leq 6 M(\delta, k) e^{-\frac{\delta^{2} n}{2 M(\delta, k)}} .
$$

Since $x e^{-t / x}$ is increasing in $x$ for $x, t>0$, we can replace $M(\delta, k)$ by any larger value $M$. 
Lemma 7. Let $x=\left[a_{1}, a_{2}, \ldots, a_{n}\right]$ and $u=\left[b_{1}, \ldots, b_{s}\right]$. Let $v$ be a block of $k$ positive integers $v_{1}, \ldots, v_{k}$. Let $\epsilon$ be a positive real less than 1 .

1. If $D_{v, n}^{c f-a r y}(x)<\epsilon$ and $D_{v, s}^{c f-a r y}(u)<\epsilon-(k-1) / s$ then $D_{v, n+s}^{c f-a r y}(x u)<\epsilon$.

2. If $D_{v, n}^{c f-a r y}(x)<\epsilon$ and $s / n<\epsilon$ then

(a) for every $\ell$ such that $1 \leq \ell \leq s, D_{v, n+\ell}^{c f-a r y}(x u)<2 \epsilon$,

(b) $D_{v, n+s}^{c f-a r y}(u x)<2 \epsilon$.

Proof. Let $\left|\left[a_{1}, \ldots, a_{n}\right]\right|_{v_{1}, \ldots, v_{k}}=\left|\left\{j: 1 \leq j \leq n-k+1, a_{j}=v_{1}, \ldots, a_{j+k-1}=v_{k}\right\}\right|$ be the number of occurrences of the block $v_{1}, \ldots, v_{k}$ in the continued fraction expansion $\left[a_{1}, \ldots, a_{n}\right]$.

For part 1, assume $D_{v, n}^{c f-a r y}(x)<\epsilon$ and $D_{v, s}^{c f-a r y}(x)<\epsilon-(k-1) / s$. Then,

$$
\begin{aligned}
|x u|_{v} & \left.\leq|x|_{v}+|u|_{v}\right)+k-1 \\
& \leq(n+s) \mu\left(I_{v}\right)+(n+s) \epsilon-s \frac{k-1}{s}+k-1 \\
& =(n+s)\left(\mu\left(I_{v}\right)+\epsilon\right) . \\
|x u|_{v} & \geq|x|_{v}+|u|_{v} \\
& \geq(n+s) \mu\left(I_{v}\right)-(n+s) \epsilon \\
& =(n+s)\left(\mu\left(I_{v}\right)-\epsilon\right) .
\end{aligned}
$$

Therefore, $D_{v, n+s}^{c f-a r y}(x u)<\epsilon$.

For part 2, assume $D_{v, n}^{c f-a r y}(x)<\epsilon$ and $s / n<\epsilon$. Let $\ell$ such that $1 \leq \ell \leq s$. Then,

$$
\begin{aligned}
\frac{\left|\left[a_{1}, \ldots, a_{n}, b_{1}, \ldots, b_{\ell}\right]\right|_{v}}{n+\ell} & \leq \frac{|x|_{v}}{n+s}+\frac{s}{n+s} \\
& \leq \frac{\left(\mu\left(I_{v}\right)+\epsilon\right) n}{n+s}+\frac{\epsilon n}{n+s} \\
& \leq\left(2 \epsilon+\mu\left(I_{v}\right)\right) \frac{n}{n+s} \\
& \leq 2 \epsilon \frac{n}{n+s}+\mu\left(I_{v}\right) \\
& \leq 2 \epsilon+\mu\left(I_{v}\right) .
\end{aligned}
$$

And

$$
\begin{array}{rlr}
\frac{\left|\left[a_{1}, \ldots, a_{n}, b_{1}, \ldots, b_{\ell}\right]\right|_{v}}{n+\ell} & \geq \frac{|x|_{v}}{n+s} \\
& \geq \frac{\left(\mu\left(I_{v}\right)-\epsilon\right) n}{n+s} \\
& \geq \mu\left(I_{v}\right)-\epsilon-\mu\left(I_{v}\right) \frac{s}{n+s} \quad \text { by elementary means } \\
& \geq \mu\left(I_{v}\right)-\epsilon-\frac{s}{n} \\
& \geq \mu\left(I_{v}\right)-2 \epsilon, \quad \text { since } \epsilon>s / n .
\end{array}
$$

We conclude $D_{v, n+\ell}^{c f-a r y}\left(\left[a_{1}, \ldots, a_{n}, b_{1} \ldots, b_{\ell}\right]\right)<2 \epsilon$.

Item $(b), D_{v, n+s}^{c f-a r y}(u x)<2 \epsilon$, is proved similarly. 


\subsection{Discrepancy associated to expansions in a given integer base}

We say that a base is an integer greater than or equal to 2 , a digit in base $b$ is an integer in $\{0, \ldots, b-1\}$, and a block in base $b$ is a finite sequence of digits in base $b$. If $u$ is a block, its length is denoted by $|u|$. We define the discrepancy of the first $n$ digits of a block $u=a_{1}, \ldots, a_{|u|}$ in base $b$ as

$$
D_{n}^{b-a r y}(u)=\max \left\{\left|\frac{1}{n} \#\left\{j: 1 \leq j \leq n, a_{j}=s\right\}-\frac{1}{b}\right|: s \in\{0, \ldots, b-1\}\right\} .
$$

Clearly, a real number $x$ is simply normal to base $b$ if and only if its expansion in base $b$, $a_{1} a_{2} \ldots$ is such that

$$
\lim _{n \rightarrow \infty} D_{n}^{b-a r y}\left(a_{1} \ldots a_{n}\right)=0 .
$$

In the construction we use the following explicit bound for the number of blocks of a given length having larger discrepancy than a given value.

Lemma 8 ([2, Lemma 2.5], adapted from [7, Theorem 148]). Fix a base $b$ and a block length $k$. For every real $\epsilon$ such that $6 / k \leq \epsilon \leq 1 / b$, the number of blocks of length $k$ with b-ary discrepancy greater than or equal to $\epsilon$ is at most $2 b^{k+1} e^{-b \epsilon^{2} k / 6}$.

If $v$ and $u$ are blocks, we write $v u$ for their concatenation.

Lemma 9 ([2, Lemma 3.1]). Let $u$ and $v$ be blocks in base $b$ and let $\epsilon>0$.

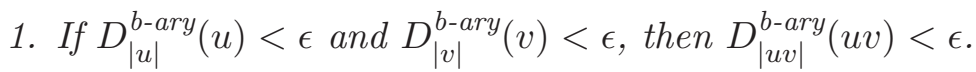

2. If $D_{|v|}^{b \text {-ary }}(v)<\epsilon$ and $|u| /|v|<\epsilon$, then

(a) for every $\ell$ less than or equal to $|u|, D_{|v|+\ell}^{b-a r y}(v u)<2 \epsilon$.

(b) $D_{|v|+|u|}^{b-a r y}(u v)<2 \epsilon$.

Proof. 1. It follows easily by considering a proper convex combination.

2. Let $\left|a_{1} \ldots a_{n}\right|_{d}=\#\left\{j: 1 \leq j \leq n, a_{j}=d\right\}$. Fix the base $b$. Let $u$ and $v$ be blocks. Fix $\ell$ and $\epsilon$. We write $(v u)_{1} \ldots(v u)_{m}$ for the block of the first $m$ digits of $(v u)$.

$$
\begin{aligned}
\frac{\left.\mid(v u)_{1} \ldots(v u)_{|v|+\ell}\right)\left.\right|_{d}}{|v|+\ell} & \geq \frac{|v|_{d}}{|v|+|u|} \\
& \geq \frac{(1 / b-\epsilon)|v|}{|v|+|u|} \\
& \geq 1 / b-\epsilon-(1 / b) \frac{|u|}{|v|+|u|}, \quad \text { by elementary means } \\
& \geq 1 / b-2 \epsilon, \quad \text { since } \epsilon>|u| /|v| .
\end{aligned}
$$

By a similar verification,

$$
\frac{\left|(v u) \ldots(v u)_{|v|+\ell}\right|_{d}}{|v|+\ell} \leq 1 / b+2 \epsilon
$$

We conclude $D_{|v|+\ell}^{b \text {-ary }}(v u)<2 \epsilon$.

The proof of point $(b), D_{|v|+|u|}^{b-a r y}(u v)<2 \epsilon$, is similar. 
As usual, for any integer $b$ greater than or equal to 2 , we say that an interval is $b$-ary, if it is of the form

$$
\left(\frac{a}{b^{k}}, \frac{a+1}{b^{k}}\right)
$$

for some positive integer $k$ and and integer $a$ with $0 \leq a<b^{k}$. In this case we also say that the interval has order $k$.

\section{Proof of Theorem 1}

The following definition is the core of the construction.

Definition 10. For an integer $t \geq 2$, a t-brick is a $t$-uple $\left(\sigma_{c f}, \sigma_{2}, \ldots, \sigma_{t}\right)$ as follows

- the interval $\sigma_{c f}$ is cf-ary;

- for every $d=2, \ldots, t, \sigma_{d}$ is d-ary interval or the union of two consecutive d-ary intervals of the same order;

- for every $d=2, \ldots, t, \sigma_{c f} \subset \sigma_{d}$ and $\left|\sigma_{c f}\right| /\left|\sigma_{d}\right| \geq 1 /\left(16 e^{4 c} d\right)$.

Definition 11. If we have a $t$-brick $\vec{\sigma}=\left(\sigma_{c f}, \sigma_{2}, \ldots, \sigma_{t}\right)$ and a $t^{\prime}$-brick $\vec{\tau}=\left(\tau_{c f}, \tau_{2}, \ldots, \tau_{t^{\prime}}\right)$ we say that $\vec{\tau}$ refines $\vec{\sigma}$ if $t^{\prime} \geq t, \tau_{c f} \subset \sigma_{c f}$ and $\tau_{d} \subset \sigma_{d}$ for $d=2, \ldots, t$. The refinement is said to have discrepancy less than $\epsilon$ if

- for each $d=2, \ldots, t$ the new block $w$ in base $d$ corresponding to the inclusion $\tau_{d} \subset \sigma_{d}$ has simple discrepancy $D^{d-a r y}(w)$ less than $\epsilon$.

- the new cf-block of digits $w$ corresponding to the inclusion $\tau_{c f} \subset \sigma_{c f}$ satisfies that for every block $v$ of $t$ digits all less than or equal to $t, D_{v,|w|}^{c f-a r y}(w)$ is less than $\epsilon-(t-1) /|w|$.

We highlight the following trivial fact about lengths of $d$-ary subintervals because it will be important in the proof of Lemma 13, our main lemma.

Proposition 12. Let $d \geq 2$ and $m \in \mathbb{N}$. Every interval $I$ whose Lebesgue measure is less than $d^{-m}$ is contained in a d-ary interval of order $m$ or in the union of two such intervals.

Lemma 13 (main lemma). Let $t$ be greater than or equal to 2, let $\epsilon$ be a positive real less than $1 / t$, and let integer $t^{\prime}$ be equal to $t$ or to $t+1$. Then, any $t$-brick $\vec{\sigma}=\left(\sigma_{c f}, \sigma_{2}, \ldots, \sigma_{t}\right)$ admits a refinement $\vec{\tau}=\left(\tau_{c f}, \tau_{2}, \ldots, \tau_{t^{\prime}}\right)$ with discrepancy less than $\epsilon$. The relative order of $\tau_{c f}$ might be any $n$ greater than certain $n_{0}(t, \epsilon)$.

Proof. First, assume $t^{\prime}=t$.

Towards the length of $\tau_{c f}$. For each $n$ consider $\mathcal{I}_{n}\left(\sigma_{c f}\right)$ the $c$-ary subintervals of $\sigma_{c f}$ of relative order $n$. Let $K, c, n_{1}$ be the constants provided by lemma 5 . Call $\mathcal{J}_{n}\left(\sigma_{c f}\right)$ the collection of intervals $J \in \mathcal{I}_{n}\left(\sigma_{c f}\right)$ such that

$$
\frac{1}{4} e^{-2 n L-2 c} \leq \frac{|J|}{\left|\sigma_{c f}\right|} \leq 2 e^{-2 n L+2 c}
$$

If $n \geq n_{1}$, lemma 5 asserts

$$
\frac{\left|\bigcup_{J \in \mathcal{J}_{n}} J\right|}{\left|\sigma_{c f}\right|} \geq \frac{K}{\sqrt{n}}
$$


At the end of the proof we will determine a value for $n$ and we will choose $\tau_{c f}$ as one of these intervals $J$ in $\mathcal{J}_{n}\left(\sigma_{c f}\right)$.

Towards the length of $\tau_{d}$. For each $n$ and for each $d=2, \ldots, t$ we call

$$
m_{d}=\operatorname{order}_{d}\left(\tau_{d}\right) .
$$

We choose $m_{d}$ as the largest integer such that $2 e^{-2 n L+2 c}\left|\sigma_{c f}\right| \leq d^{-m_{d}}$. This choice guarantees $|J| \leq d^{-m_{d}}$ for every $J \in \mathcal{J}_{n}$ and also

$$
d^{-m_{d}-1} \leq 2 e^{-2 n L+2 c}\left|\sigma_{c f}\right|=8 e^{4 c} \frac{1}{4} e^{-2 n L-2 c}\left|\sigma_{c f}\right| \leq 8 e^{4 c}|J| .
$$

For each $J \in \mathcal{J}_{n}$ we determine $\tau_{d}^{J}$ as the $d$-ary interval of order $m_{d}$ or the union of two consecutive $d$-ary intervals of order $m_{d}$ that contain $J$ (Proposition 12). Thus,

$$
\frac{|J|}{\left|\tau_{d}^{J}\right|} \geq \frac{1}{16 e^{4 c} d}
$$

This choice of $m_{d}$ imposes bounds on $n_{d}=\operatorname{order}\left(\tau_{d}\right)-\operatorname{order}\left(\sigma_{d}\right)$ which only depend on $n$ and $d$, as follows:

1. Since $\left|\sigma_{c f}\right| \leq\left|\sigma_{d}\right| \leq\left|\sigma_{c f}\right| 16 e^{4 c} d$ then

$$
\log _{d}\left(\left|\sigma_{c f}\right| / 2\right) \leq-\operatorname{order}\left(\sigma_{d}\right) \leq \log _{d}\left(\left|\sigma_{c f}\right| 16 e^{4 c} d\right) .
$$

Notice that $\operatorname{order}\left(\sigma_{d}\right)=-\log _{d}\left(\left|\sigma_{d}\right| / 2\right)$ or $\operatorname{order}\left(\sigma_{d}\right)=-\log _{d}\left(\left|\sigma_{d}\right|\right)$.

2. And since $2 e^{-2 n L+2 c}\left|\sigma_{c f}\right| \leq d^{-m_{d}} \leq d 2 e^{-2 n L+2 c}\left|\sigma_{c f}\right|$ then

$$
\log _{d}\left(2 e^{-2 n L+2 c}\left|\sigma_{c f}\right|\right) \leq-\operatorname{order}\left(\tau_{d}\right)=-m_{d} \leq \log _{d}\left(d 2 e^{-2 n L+2 c}\left|\sigma_{c f}\right|\right) .
$$

We obtain,

$$
2 n L \log _{d} e-\log _{d}\left(4 d e^{2 c}\right) \leq \operatorname{order}\left(\tau_{d}\right)-\operatorname{order}\left(\sigma_{d}\right) \leq 2 n L \log _{d} e+\log _{d}\left(8 e^{2 c} d\right)
$$

Then, since $n_{d}=\operatorname{order}\left(\tau_{d}\right)-\operatorname{order}\left(\sigma_{d}\right)$,

$$
2 n \frac{L}{\log d}-\frac{2 c}{\log d}-3 \leq n_{d} \leq 2 n \frac{L}{\log d}+\frac{2 c}{\log d}+4 .
$$

Bad zones. We must pick one interval $J$ in $\mathcal{J}_{n}$ in a zone of low discrepancy. This is possible because the measure of the zones of large discrepancy decrease at an exponential rate in $n$ while the measure of $\mathcal{J}_{n}$ decreases only as $K / \sqrt{n}$.

For each $n$ let

$$
B_{d, \sigma_{d}, m_{d}, \epsilon}^{0}
$$

be the set of reals in the $d$-ary subintervals of $\sigma_{d}$ of order $m_{d}$ with $d$-discrepancy greater than $\epsilon$. And let

$$
B_{d, \sigma_{d}, m_{d}, \epsilon}
$$

be the union of $B_{d, \sigma_{d}, m_{d}, \epsilon}^{0}$ with those numbers lying in a $d$-ary interval of the same order that is a neighbour to one in $B_{d, \sigma_{d}, m_{d}, \epsilon}^{0}$. 
With the conditions $6 / n_{d} \leq \epsilon \leq 1 / d$, Lemma 8 gives the estimate

$$
\frac{\left|B_{d, \sigma_{d}, m_{d}, \epsilon}\right|}{\left|\sigma_{d}\right|} \leq 6 d e^{-d \epsilon^{2} n_{d} / 6}
$$

Since $n_{d} \geq 2 n \frac{L}{\log d}-\frac{2 c}{\log d}-3$, and $\left|\sigma_{d}\right| \leq 16 e^{4 c} d\left|\sigma_{c f}\right|$, we have

$$
\begin{aligned}
\frac{\left|B_{d, \sigma_{d}, m_{d}, \epsilon}\right|}{\left|\sigma_{c f}\right|} & \leq 16 e^{4 c} d \frac{\left|B_{d, \sigma_{d}, m_{d}, \epsilon}\right|}{\left|\sigma_{d}\right|} \\
& \leq 96 e^{4 c} d^{2} e^{-d \epsilon^{2} n_{d} / 6} \\
& \leq A(d) e^{-d \epsilon^{2} L n /(3 \log d)}
\end{aligned}
$$

where $A(d)=96 e^{4 c} d^{2} e^{d \epsilon^{2}(c /(3 \log d)+1 / 2)}$.

For each $n$, let

$$
\tilde{B}_{t, \sigma_{c f}, n, \epsilon}
$$

be the set of reals $x$ in the $c$-ary subintervals of $\sigma_{c f}$ of relative order $n$ such that for some block of length $t$ of digits less than or equal to $t$ the $c f$-discrepancy of $x$ is greater than $\epsilon-(t-1) / n$. With the condition $2(t-1) / \epsilon \leq n$, it suffices to consider $c f$-discrepancy greater than $\epsilon / 2$. Lemma 6 gives the estimate,

$$
\frac{\left|\tilde{B}_{t, \sigma_{c f}, n, \epsilon}\right|}{\left|\sigma_{c f}\right|} \leq t^{t} 6 M e^{-\frac{(\epsilon / 2)^{2} n}{2 M}}
$$

where $M=\left\lceil t-\log \left(\frac{(\epsilon / 2)^{2}}{2 \log 2}\right)\right\rceil$ or larger.

Find $n_{0}$ large enough. Now we choose $n_{0}$ such that for $n \geq n_{0}$ the bad zones are smaller than the measure of the union of $\mathcal{J}_{n}$. We need a value of $n$ such that

$$
\begin{aligned}
6 M t^{t} e^{-\frac{(\epsilon / 2)^{2} n}{2 M}} & \leq \frac{K}{t \sqrt{n}}, \quad \text { and for } d=2, \ldots, t, \\
A(d) e^{-d \epsilon^{2} L n /(3 \log d)} & \leq \frac{K}{t \sqrt{n}} .
\end{aligned}
$$

Hence, we need to find solutions to

$$
\sqrt{n} e^{-r n} \leq \gamma
$$

for certain values of $r$ and $\gamma$. Since for every positive $x$, it holds that $x<e^{x / 2}$, we have

$$
\sqrt{n} e^{-r n / 2} \leq \frac{1}{r} r n e^{-r n / 2}<\frac{1}{r} e^{r n / 2-r n / 2}=\frac{1}{r} .
$$

Thus, we need $n_{0}$ such that

$$
e^{-r n / 2} \leq \gamma r
$$

for each of the needed values $r$ and $\gamma$. Hence, $n_{0}$ has to be as large as

$$
-2 / r \log (\gamma r) .
$$

for each of the needed values $r$ and $\gamma$. 


\section{Letting}

$$
\begin{array}{ll}
r^{(1)}=\varepsilon^{2} /(8 M), & \gamma^{(1)}=K /\left(6 M t^{t+1}\right), \\
r^{(d)}=d \varepsilon^{2} L /(3 \log d), & \gamma^{(d)}=K /(t A(d)) .
\end{array} \quad \text { and for } d=2, \ldots, t,
$$

we have to take

$$
n_{0}=\max \left\{-2 / r^{(d)} \log \left(\gamma^{(d)} r^{(d)}\right): 1 \leq d \leq t\right\} \cup\left\{\frac{6}{\epsilon}, \frac{2(t-1)}{\epsilon}, n_{1}\right\}
$$

This completes the proof in case $t^{\prime}=t$.

The case $t^{\prime}=t+1$ follows easily by taking first a $t$-brick $\vec{\tau}$ refining $\vec{\sigma}$ with discrepancy less than $\epsilon$. Then we only need to take $\tau_{t+1}$ a $(t+1)$-ary interval of order $m_{t+1}$, or a union of two consecutive such intervals so that $\tau_{c f} \subset \tau_{t+1}$ and $\tau_{t+1}$ not very large. For instance, we can take $m_{t+1}$ to be the maximum such that $\left|\tau_{c f}\right| \leq(t+1)^{-m_{t+1}}$, so that applying again Proposition 12,

$$
\frac{\left|\tau_{c f}\right|}{\left|\tau_{t+1}\right|} \geq \frac{1}{2(t+1)}
$$

\subsection{Algorithm}

The algorithm constructs a sequence of $t$-bricks $\vec{\sigma}_{1}, \vec{\sigma}_{2}, \vec{\sigma}_{3}, \ldots$ for non-decreasing values of $t$. The real number defined by the intersection of all the intervals in the sequence is absolutely normal and continued fraction normal.

We consider the block length $t$, the discrepancy value $\epsilon$ and the relative order $n$ of the new $c f$-ary interval as functions of the step $s$. Define for every positive $s$

$$
\begin{aligned}
& t(s)=\max (2,\lfloor\sqrt[5]{\log s}\rfloor), \\
& \epsilon(s)=1 / t(s) .
\end{aligned}
$$

Clearly $t(s)$ is non-decreasing unbounded and $\epsilon(s)$ is non-increasing and goes to zero. Now consider the function $n_{0}(\epsilon(s), t(s))$ given by Lemma 13 and notice that

$$
n_{0}(\epsilon(s), t(s)) \text { is in } O\left(t(s)^{4} \log (t(s))\right) \text {. }
$$

Let $n_{\text {start }}$ be the minimum positive integer such that for every positive $s$

$$
\lfloor\log s\rfloor+n_{\text {start }} \geq n_{0}(\epsilon(s), t(s))
$$

and define

$$
n(s)=\lfloor\log s\rfloor+n_{\text {start }} .
$$

The algorithm is as follows.

Initial step, $s=1$. Let $\vec{\sigma}_{1}=\left(\sigma_{c f}, \sigma_{2}\right)$, with $\sigma_{2}=\sigma_{c f}=(0,1)$.

Recursive step, $s>1$. Assume $\vec{\sigma}_{s-1}=\left(\sigma_{c f}, \sigma_{2}, \ldots, \sigma_{t(s-1)}\right)$. Take $\vec{\sigma}_{s}=\left(\tau_{c f}, \tau_{2}, \ldots, \tau_{t(s)}\right)$ the leftmost refinement of $\vec{\sigma}_{s-1}$ with discrepancy less than $\epsilon(s)$ and such that the order of $\tau_{c f}$ is $n(s)$ plus the order of $\sigma_{c f}$. 


\subsection{Correctness}

The existence of the sequence $\vec{\sigma}_{1}, \vec{\sigma}_{2}, \ldots$ is guaranteed by Lemma 13. We have to prove that the real number $x$ defined by the intersection of all the intervals in the sequence is absolutely normal and continued fraction normal.

We first show that $x$ has a normal continued fraction expansion. Let $v$ be a block of $m$ integers $v_{1}, \ldots, v_{m}$ and let $\tilde{\epsilon}>0$. Choose $s_{0}$ so that $m \leq t\left(s_{0}\right), \max \left\{v_{1}, \ldots, v_{m}\right\} \leq t\left(s_{0}\right)$ and $\epsilon\left(s_{0}\right) \leq \tilde{\epsilon} / 4$. At each step $s$ after $s_{0}$, the continued fraction expansion of $x$ is constructed by appending a block $u_{s}$ such that $\left|u_{s}\right|=n(s)$ and

$$
D_{v,\left|u_{s}\right|}^{c f-a r y}\left(u_{s}\right)<\epsilon(s)-\frac{t(s-1)-1}{\left|u_{s}\right|}<\epsilon(s)-\frac{m-1}{\left|u_{s}\right|} .
$$

By Lemma 7 (item 1) applied many times, for every $s \geq s_{0}$ :

$$
D_{v,\left|u_{s_{0}} \ldots u_{s}\right|}^{c f-a r y}\left(u_{s_{0}} u_{s_{0}+1} \ldots u_{s}\right)<\epsilon\left(s_{0}\right) .
$$

Next, by Lemma 7 (item $2 \mathrm{~b}$ ) there is $s_{1}$ sufficiently large such that for every $s \geq s_{1}$,

$$
D_{v,\left|u_{1} \ldots u_{s}\right|}^{c f-a r y}\left(u_{1} \ldots u_{s}\right)<2 \epsilon\left(s_{0}\right) .
$$

Since $n(s)$ grows logarithmically, the inequality

$$
n(s) \leq 2 \epsilon\left(s_{0}\right) \sum_{j=1}^{s-1} n(j)
$$

holds from certain point on. Hence, by Lemma 7 (item 2a), we have for every $s$ sufficiently large and for every $\ell$ such that $\left|u_{1} \ldots u_{s-1}\right|<\ell \leq\left|u_{1} \ldots u_{s}\right|$,

$$
D_{v, \ell}^{c f-a r y}\left(u_{1} \ldots u_{s}\right)<4 \epsilon\left(s_{0}\right)<\tilde{\epsilon} .
$$

It follows that $x$ is continued fraction normal.

The argument to show that $x$ is absolutely normal is very similar. We pick a base $d$ and show that $x$ is simply normal to base $d$. Let $\tilde{\epsilon}>0$. Choose $s_{0}$ so that $t\left(s_{0}\right) \geq d$ and $\epsilon\left(s_{0}\right) \leq \tilde{\epsilon} / 4$. At each step $s$ after $s_{0}$ the expansion of $x$ in base $d$ was constructed by appending blocks $u_{s}$ such that $D_{\left|u_{s}\right|}^{d \text { ary }}\left(u_{s}\right)<\epsilon\left(s_{0}\right)$. Thus, by Lemma 9 (item 1 ) for any $s>s_{0}$,

$$
D_{\mid u_{s_{0} \ldots u_{s} \mid}}^{d-a r y}\left(u_{s_{0}} \ldots u_{s}\right)<\epsilon\left(s_{0}\right) .
$$

Applying Lemma 9 (item 2a), we obtain $s_{1}$ such that for any $s>s_{1}$

$$
D_{\left|u_{1} \ldots u_{s}\right|}^{d-a r y}\left(u_{1} \ldots u_{s}\right)<2 \epsilon\left(s_{0}\right) .
$$

Call $n_{d}(j)$ the relative order of the $d$-interval of $\vec{\sigma}_{n(j)}$ with respect to the $d$-interval of $\vec{\sigma}_{n(j-1)}$. The inequalities

$$
2 n(j) \frac{L}{\log d}-\frac{2 c}{\log d}-3 \leq n_{d}(j) \leq 2 n(j) \frac{L}{\log d}+\frac{2 c}{\log d}+4
$$

provided by the proof of Lemma 13, tell us that $n_{d}(j)$ grows logarithmically. 
Thus, for $s$ sufficiently large we have

$$
n_{d}(s) \leq 2 \epsilon\left(s_{0}\right) \sum_{j=1}^{s-1} n_{d}(j) .
$$

By Lemma 9 (item 2b) we conclude that for $s$ sufficiently large and $\left|u_{1} \ldots u_{s-1}\right| \leq \ell \leq\left|u_{1} \ldots u_{s}\right|$,

$$
D_{\ell}^{d-a r y}\left(u_{1} \ldots u_{s}\right)<4 \epsilon\left(s_{0}\right)<\tilde{\epsilon} .
$$

So, $x$ is simply normal to base $d$ for every $d \geq 2$.

\subsection{Computational complexity}

We analyse the computational complexity of the algorithm described in the previous section by counting the number of mathematical operations required to output the first $k$ digits of the continued fraction expansion of the computed number. We would obtain an equivalent outcome if we counted the number of mathematical operations required to output the first $k$ digits of the expansion of the computed number in some prescribed base. Here we do not count how many elementary operations are implied by each of the mathematical operations, which means that we neglect the computational cost of performing arithmetical operations with arbitrary precision.

Memory assumptions at step s. Let $N(s)=\sum_{i=1}^{s} n(i)$. At the beginning of step $s$ the current $t$-brick is $\vec{\sigma}_{s-1}=\left(\sigma_{c f}, \sigma_{2}, \ldots, \sigma_{t(s-1)}\right)$. Let $z_{c f}$ be the left endpoint of $\sigma_{c f}$ and let $\left[a_{1}, \ldots, a_{N(s-1)}\right]$ be is continued fraction expansion. Let $z_{d}$ be the left endpoint of $\sigma_{d}$. We assume that at step $s$ the algorithm has direct access to the following values:

1. the approximant $p_{N(s-1)} / q_{N(s-1)}$ of $\left[a_{1}, \ldots, a_{N(s-1)}\right]$. In this way, the algorithm has access to the value $z_{c f}$,

2. the value $\left|\sigma_{c f}\right|$,

3. the values $z_{c f}-z_{d}$, for $d=2, \ldots, t(s)$

We do not require direct access to any other values.

How to pick a t-brick in the good zone at step s. Lemma 13 ensures the existence of the wanted $t$-brick . To effectively find it we proceed as follows. Divide the interval $\sigma_{c f}$ into

$$
\left\lfloor 4 e^{2 n(s) L+2 c}\right\rfloor+1
$$

equal intervals. Notice that every interval contained in $\sigma_{c f}$ whose length is at least

$$
\frac{1}{4} e^{-2 n(s) L-2 c}\left|\sigma_{c f}\right|
$$

will contain as interior point an endpoint of these equal intervals. For each endpoint determine if it belongs to a $c f$-ary subinterval $J_{c f}$ of $\sigma_{c f}$ of relative order $n(s)$ whose length is between

$$
\frac{1}{4} e^{-2 n(s) L-2 c}\left|\sigma_{c f}\right| \text { and } 2 e^{2 n(s) L+2 c}\left|\sigma_{c f}\right| .
$$


In case it does, determine if the corresponding $t$-brick $\vec{J}=\left(J_{c f}, J_{2}, \ldots, J_{t(s)}\right)$ refines $\vec{\sigma}_{s-1}$ with discrepancy less than $\epsilon$. For this we need to determine the blocks that lead from the intervals $\sigma_{c f}, \sigma_{2}, \ldots, \sigma_{t(s-1)}$ to the intervals $J_{c f}, J_{2}, \ldots, J_{t(s-1)}$. Thus, given $\sigma_{c f}$, we need to determine the block of $n(s)$ many digits that leads to $J_{c f}$. And for $d=2, \ldots, t(s-1)$, given $\sigma_{d}$, we need to determine the block of $n_{d}=\operatorname{order}\left(J_{d}\right)-\operatorname{order}\left(\sigma_{d}\right)$ many digits in base $d$ that leads to $J_{d}$.

An upper bound for the number of mathematical operations at step s. In the worst case, to find a wanted $t$-brick we have to inspect all the candidate endpoints. Since $n(s)=\lfloor\log s\rfloor+n_{\text {start }}$, the total number $T$ of candidate endpoints is

$$
\left\lfloor 4 e^{2\left(\lfloor\log s\rfloor+n_{\text {start }}\right) L+2 c}\right\rfloor .
$$

Thus, the number of endpoints is in

$$
O\left(s^{2 L}\right) \text {. }
$$

Let $e_{0}, \ldots e_{T-1}$ be these endpoints. We write each endpoint $e_{j}$, for $j=0, \ldots, T-1$, as

$$
e_{j}=z_{c f}+\left|\sigma_{c f}\right| j / T \text {. }
$$

Let $u, v$ be integers such that $\left|\sigma_{c f}\right| j / T=u / v$. Then the continued fraction expansion of $e_{j}$ can be written as $\left[a_{1}, \ldots, a_{N(s-1)}\right]$ concatenated with the continued fraction expansion of $u / v$. We only need $n(s)$ many digits of continued fraction expansion of $u / v$ that we can obtain by running the Euclidean algorithm on $(u, v)$ for $n(s)$ iterations. This gives a number of mathematical operations in

$$
O(n(s)) \text {. }
$$

Let $J_{c f}$ be the $c f$-ary subinterval of $\sigma_{c f}$ of relative order $n(s)$. The computation of its length requires computing the convergents $q_{N(s-1)+1}, \ldots, q_{N(s-1)+n(s)}$. Thus checking that the length is suitable requires a number of mathematical operations in

$$
O(n(s)) \text {. }
$$

Now we write each endpoint $e_{j}$, for $j=0, \ldots, T-1$, as

$$
e_{j}=\left(z_{c f}-z_{d}\right)+z_{d}+\left|\sigma_{c f}\right| j / T .
$$

Then, the base- $d$ expansion of $e_{j}$ consists of the base- $d$ expansion of $z_{d}$ followed by the base $d$-expansion of $\left(z_{c f}-z_{d}\right)+\left|\sigma_{c f}\right| j / T$. By the proof of Lemma 13, for each base $d$, we just need $n_{d}$ many digits of this expansion and $n_{d}$ is $O(n(s))$. The conversion of the rational value $\left(z_{c f}-z_{d}\right)+\left|\sigma_{c f}\right| j / T$ to base $d$ can be done by a constant number of mathematical operations.

Finally, we need to check if the discrepancy of each of the $t$ blocks witnessed by $e_{j}$ is less than $\epsilon(s)$. This can be done by a number of comparisons that is linear in the length of the block plus a constant number of operations, hence in

$$
O(n(s)) \text {. }
$$

We conclude that at step $s$ in the worst case the number of required mathematical operations to choose $\vec{\sigma}_{s}$ can be bounded as

$$
O(T(n(s)+n(s)+t(s) \text { constant }+t(s) n(s))) .
$$


Since $T$ is in $O\left(s^{2 L}\right), n(s)$ is in $O(\log (s))$, and $t(s)$ is in $O\left(\log ^{1 / 5}(s)\right)$, the total number of mathematical operations at step $s$ is

$$
O\left(s^{2 L} \log ^{6 / 5}(s)\right) .
$$

Number of mathematical operations to compute the first $k$ digits. After the first $k$ steps the number of digits of the continued fraction expansion of $x$ obtained by the algorithm is $N(k)$, which is greater than $k$. While the number of mathematical operations performed by the algorithm is in the order of

$$
\sum_{s=1}^{k} s^{2 L} \log ^{6 / 5}(s) \text { which is less than } k^{2 L+1} \log ^{6 / 5}(k),
$$

and this last expesion is in $O\left(k^{4}\right)$. This completes the proof of Theorem 1

Acknowledgments. We are thankful to Brigitte Vallée for pointing to us the optimal version of the Central Limit Theorem that establishes that the distribution of the logarithm of the convergents of finite continued fractions is asymptotically Gaussian, as well as its implication on the length of $c f$-intervals. Both authors are supported by grant PICT 2014-3260 by Agencia Nacional de Promoción Científica y Tecnológica, Argentina. Becher is a member of Laboratoire International Associé INFINIS, Université Paris Diderot-CNRS / Universidad de Buenos Aires-CONICET). Becher worked towards this paper at the Erwin Schrödinger International Institute for Mathematics and Physics, Austria.

\section{References}

[1] R. Adler, M. Keane, and M. Smorodinsky. A construction of a normal number for the continued fraction transformation. Journal of Number Theory, 13(1):95 - 105, 1981.

[2] V. Becher, P.A. Heiber, and T. Slaman. A polynomial-time algorithm for computing absolutely normal numbers. Information and Computation, 232:1-9, 2013.

[3] G.D. Birkhoff. Proof of the Ergodic Theorem. Proc. Nat. Acad. Sci., 17:656-660, 1931.

[4] É. Borel. Les probabilités d'enombrables et leurs applications arithmétiques. Supplemento di Rendiconti del Circolo Matematico di Palermo, 27:247-271, 1909.

[5] Y. Bugeaud. Distribution Modulo One and Diophantine Approximation. Number 193 in Cambridge Tracts in Mathematics. Cambridge University Press, Cambridge, UK, 2012.

[6] P. Flajolet and B. Vallée. Continued fraction algorithms, functional operators, and structure constants. Theoretical Computer Science, 194(1):1 - 34, 1998. Theorem 1.

[7] G. H. Hardy and E. M. Wright. An introduction to the theory of numbers. Oxford University Press, Oxford, sixth edition, 2008.

[8] I. Ibargimov. A theorem from the metric theory of continued fractions. Vestnik Leningrad. Univ., 16(1):13-24, 1961. 
[9] Y. Kifer, Y. Peres, and B Weiss. A dimension gap for continued fractions with independent digits. Israel Journal of Mathematics, 124(1):61-76, 2001.

[10] M. Madritsch, A.-M.Scheerer, and R. Tichy. Computable absolutely nrmal pisot numbers. arXiv:1610.06388v1, 2017.

[11] M.G. Madritsch and B. Mance. Construction of $\mu$-normal sequences. Monatshefte für Mathematik, 179(2):259-280, 2016.

[12] G.A. Mischyavichyus. Estimate of the remainder in the limit theorem for the denominators of continued fractions. Litovskii Matematiceskii Sbomik, 21(3):63-74, 1987.

[13] T. Morita. Local limit theorem and distribution of periodic orbits of Lasota-Yorke transformations with infinite Markov partitions. J. Math. Sot. Japan, 46(2):309-343, 1994. Corrections in Vol. 47 (I) (1997) 191-192.

[14] W. Philipp. Ein zentraler grenzwertsatz mit anwendungen auf die zahlentheorie. Wahrscheinlichkeitstheorie verw. Geb., 8:185-203, 1967. Theorem 3.

[15] A. G. Postnikov and I. I. Pyatetskii. A Markov-sequence of symbols and a normal continued fraction. Izv. Akad. Nauk SSSR Ser. Mat., 21(6):729-746, 1957.

[16] M. Quéffelec. Old and new results on normality. In Dynamics and Stochastics, IMS Lecture Notes Monogr. Ser., 48, pages 225-236. Institute of Mathematics and Statistics, Beachwood, OH, 2006.

[17] A.-M. Scheerer. On the continued fraction expansion of absolutely normal numbers. arXiv:1701.07979v1, 2017.

[18] B. Vallée. Operateurs de Ruelle-Mayer generalises et analyse des algorithmes d'Euclide et de Gauss. Acta Arithmetica, LXXXI.2:101-144, 1997. values for the constants.

Verónica Becher

Departmento de Computación, Facultad de Ciencias Exactas y Naturales

Universidad de Buenos Aires \& ICC CONICET, Argentina.

vbecher@dc.uba.ar

Sergio Yuhjtman

Departmento de Matemática, Facultad de Ciencias Exactas y Naturales

Universidad de Buenos Aires, Argentina.

syuhjtma@dm.uba.ar 